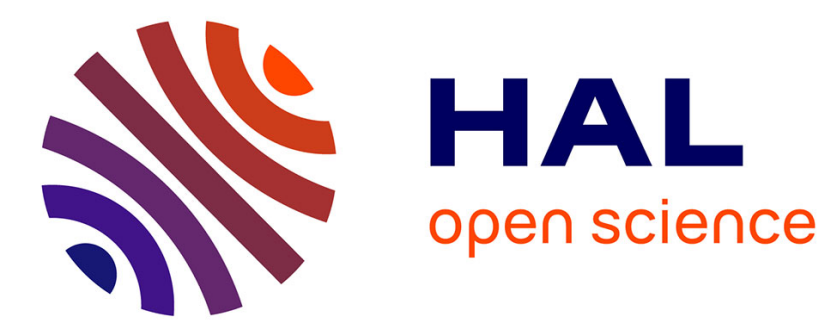

\title{
Prendre en compte la diversité des élèves au collège : entre discours et pratiques des enseignants-stagiaires en Institut Universitaire de Formation des Maîtres
}

Thérèse Perez-Roux

\section{- To cite this version:}

Thérèse Perez-Roux. Prendre en compte la diversité des élèves au collège : entre discours et pratiques des enseignants-stagiaires en Institut Universitaire de Formation des Maîtres. Les Sciences de l'éducation pour l'ère nouvelle: revue internationale, 2007, 40 (4), pp.107 - 134 . 10.3917/lsdle.404.0107 . hal-01716185

\section{HAL Id: hal-01716185 \\ https://hal.science/hal-01716185}

Submitted on 23 Feb 2018

HAL is a multi-disciplinary open access archive for the deposit and dissemination of scientific research documents, whether they are published or not. The documents may come from teaching and research institutions in France or abroad, or from public or private research centers.
L'archive ouverte pluridisciplinaire HAL, est destinée au dépôt et à la diffusion de documents scientifiques de niveau recherche, publiés ou non, émanant des établissements d'enseignement et de recherche français ou étrangers, des laboratoires publics ou privés. 


\section{PRENDRE EN COMPTE LA DIVERSITÉ DES ÉLÈVES AU COLLÈGE : ENTRE DISCOURS ET PRATIQUES DES ENSEIGNANTS-STAGIAIRES EN INSTITUT UNIVERSITAIRE DE FORMATION DES MAÎTRES} Thérèse Perez-Roux

CERSE - Université de Caen | «Les Sciences de l'éducation - Pour l'Ėre nouvelle » 2007/4 Vol. 40 | pages 107 à 134

ISSN 0755-9593

ISBN 9782952802543

Article disponible en ligne à l'adresse :

https://www.cairn.info/revue-les-sciences-de-l-education-pour-l-erenouvelle-2007-4-page-107.htm

\section{Pour citer cet article :}

Thérèse Perez-Roux, « Prendre en compte la diversité des élèves au collège : entre discours et pratiques des enseignants-stagiaires en Institut Universitaire de Formation des Maîtres », Les Sciences de l'éducation - Pour l'Ėre nouvelle 2007/4 (Vol. 40), p. 107-134.

DOI 10.3917/lsdle.404.0107

Distribution électronique Cairn.info pour CERSE - Université de Caen.

(C) CERSE - Université de Caen. Tous droits réservés pour tous pays.

La reproduction ou représentation de cet article, notamment par photocopie, n'est autorisée que dans les limites des conditions générales d'utilisation du site ou, le cas échéant, des conditions générales de la licence souscrite par votre établissement. Toute autre reproduction ou représentation, en tout ou partie, sous quelque forme et de quelque manière que ce soit, est interdite sauf accord préalable et écrit de l'éditeur, en dehors des cas prévus par la législation en vigueur en France. Il est précisé que son stockage dans une base de données est également interdit. 


\section{Prendre en compte la diversité des élèves au collège : entre discours et pratiques des enseignants-stagiaires en Institut Universitaire de Formation des Maîtres}

Thérèse PEREZ-Roux*

Résumé : Durant la deuxième année de formation à l'Institut Universitaire de Formation des Maîtres (IUFM), les professeurs-stagiaires effectuent un stage en responsabilité dans un établissement scolaire. Létude se centre sur les processus de construction de l'identité professionnelle des enseignants débutants, notamment lorsque le collège les confronte à l'hétérogénéité des élèves en matière d'apprentissage. Elle s'intéresse aux écarts entre normes de la formation, représentations sur le métier et pratiques professionnelles.

Un premier recueil de données, par questionnaire, est complété par trois entretiens semi-directifs réalisés dans l'année 2004-2005 avec six stagiaires du corpus, observés par ailleurs lors de séances effectives. Au-delà de la manière dont le groupe professionnel se situe par rapport à la diversité des publics scolaires, une étude de cas permet de repérer des solutions envisagées localement, dans l'ordinaire de la classe. Ces réponses rendent compte d'adaptations provisoires; elles contribuent au développement d'une identité professionnelle tributaire des relations entre logique des acteurs, logique de formation et contexte de travail.

Mots-clés : Identité professionnelle. Formation. Logiques d'action. Pratiques.

*Maître de Conférences en Sciences de l'éducation, IUFM des Pays de Loire, Laboratoire du CREN, Université de Nantes. 


\section{Introduction}

\subsection{Contexte de l'étude}

Dans une dynamique de professionnalisation progressive, la deuxième année à l'IUFM, pensée sur le mode de l'alternance, place la pratique professionnelle au cœur du dispositif de formation. Dans ce cadre, chaque enseignant-stagiaire ${ }^{1}$ est invité à articuler expérience pratique et acquisition de savoirs professionnels en vue de construire des compétences professionnelles multiples.

Notre recherche porte sur les processus de construction de l'identité professionnelle des stagiaires en formation à l'IUFM, notamment lorsque le collège les confronte à la diversité des élèves en matière d'apprentissage. En effet, devant les missions parfois contradictoires assignées à ce niveau du cursus scolaire (accueillir, instruire, socialiser, orienter, etc.), les enseignants sont soumis à de multiples tensions et doivent trouver les moyens d'y faire face quotidiennement. Dans la mesure où l'hétérogénéité des élèves et la complexité des situations d'enseignement sont perçues de façon plus vive en début de carrière (HeTU, LaVOIE \& BaILLAUQUÈs, 1999; RAYOU \& VAN ZANTEN, 2004), l'étude tente de repérer les points d'appui mobilisés durant l'année de formation, les tensions et adaptations conduisant à l'appropriation progressive de compétences professionnelles, envisagées comme un élément majeur dans le processus de construction identitaire (BARBIER, 1996; DUBAR, 1991; LAUTIER, 2001). Elle s'intéresse plus particulièrement à la manière dont les stagiaires construisent leur monde professionnel. Il s'agit de mettre en relief un certain nombre de représentations permettant à ces derniers de fonder, justifier et rationaliser leur pratique professionnelle, lorsqu'ils rencontrent la réalité du collège et les enjeux de formation qui le traversent.

\subsection{Représentations, savoirs et compétences au cœur du processus de professionnalisation}

L'entrée dans le métier s'effectue sur la base de représentations fondées sur des valeurs mais aussi des discours, des cultures, des projections ancrées dans l'expérience scolaire (BAILLAUQUÈs, 1996). Ces représentations donnent sens à la pratique et légitiment une certaine «vision du monde» (MOSCOVICI, 1961; JODELET, 1984). Elles servent à agir

1. Pour ne pas alourdir le texte, nous remplacerons le terme «enseignant-stagiaire» par celui de «stagiaire» dans la suite de l'article. 
et réagir face à l'environnement tout en conservant un équilibre cognitif dans un contexte professionnel particulier.

À partir de cet ensemble d'éléments, s'élabore une conception de «l'enseignant idéal», auquel chacun tente de se référer tout en faisant en sorte de réduire les écarts avec la réalité de la pratique professionnelle. Mais ce processus, loin d'être linéaire, suppose des moments de remise en cause des représentations initiales; il s'agit alors de les dépasser pour construire de nouvelles cohérences, nécessaires au développement des compétences professionnelles.

Par ailleurs, en mettant en évidence un système de normes, le processus de professionnalisation invite l'enseignant à revisiter les premières conceptions de l'enseignement et à construire de nouveaux savoirs, pluriels et combinés dans l'action. Les compétences professionnelles intègrent un certain nombre d'entre eux, formant une totalité complexe, mouvante, structurée, opératoire, c'est-à-dire ajustée à l'action (MALGLAIVE, 1990). Ces savoirs «se combinent dans des représentations et des théories personnelles qui sont réinvesties par la personne dans l'action» (CHARLIER, 1996, 104).

En ce sens, les représentations sont des matrices d'action constitutives des identités professionnelles (BARBIER, 1996; Blin, 1997; Dubar, 1991; RouX-PEREZ, 2005 (a); TAP, 1998).

\subsection{Des identités professionnelles en construction}

Dans cette perspective, l'identité professionnelle est envisagée dans une double transaction biographique et relationnelle (DUBAR, 1992). Ce moment crucial de l'entrée dans le métier enseignant implique des changements auxquels le stagiaire est tenu de s'adapter. Comment préserve-t-il le sentiment de rester le même (de conserver ses valeurs) tout en se transformant pour faire face à la complexité du métier? Par ailleurs, chacun élabore une «image de soi» en relation - accord, tension, contradiction avec celles que, selon lui, les autres lui attribuent : cela conduit à un sentiment de reconnaissance ou de non reconnaissance d'autrui essentiel dans la construction identitaire. Comment le stagiaire prend-il en compte l'ensemble des regards croisés sur sa pratique lors de l'année de stage? Dans quelle mesure cela l'aide-t-il à se définir comme professionnel? Face à un ensemble de transactions envisagées comme des actions qui traversent la situation individuelle et nécessitent délibérations, ajustements et compromis, l'enseignant cherche à construire «un monde qui tient» (DEROUET, 1992). Pour trouver un équilibre, il se représente la discipline, le métier, les pratiques professionnelles et s'engage dans l'action (RouX-PEREZ, 2003). Le degré de reconnaissance d'autrui et le sentiment de contrôle de la situation qui en résultent peuvent conduire à une réorganisation partielle des représentations et des registres d'action. Au-delà 
des inévitables tensions liées à l'entrée dans le métier, quelles pratiques professionnelles sont mises en œuvre? Comment les stagiaires s'organisent-ils entre normes de la formation, valeurs personnelles et contraintes de la situation de classe, dans un contexte et une discipline dont ils saisissent progressivement les enjeux et les limites?

Après avoir précisé les aspects méthodologiques choisis pour l'étude, nous tenterons de répondre à ces questions avec deux entrées complémentaires; un premier niveau de résultats tente d'éclairer la manière dont le groupe professionnel se situe par rapport à la diversité des publics scolaires. Dans un deuxième temps, une étude de cas permet de repérer : 1) les éventuels écarts entre valeurs, représentations professionnelles et réalité du contexte d'enseignement; 2) les transactions à l'œuvre pour favoriser régulations et prises de repères durant l'année de professionnalisation; 3) les formes de pratiques qui en résultent, notamment face à la diversité des élèves en matière d'apprentissage, et les effets en termes de construction identitaire.

\section{Méthodologie}

Une enquête par questionnaire a été proposée en novembre 2004 à 181 enseignants-stagiaires des Pays de la Loire effectuant leur stage en responsabilité au collège ${ }^{2}$. Les 141 questionnaires retournés, analysés avec le logiciel Sphinx, donnent une vision générale du groupe et mettent en relief des spécificités liées au genre, aux expériences antérieures, à la discipline enseignée et au contexte du stage en responsabilité (ROUX-PEREZ, 2006 (a)).

Dans le prolongement de cette première enquête, treize stagiaires ont accepté de s'inscrire dans un dispositif de recherche résolument qualitatif. Trois entretiens semidirectifs ont été conduits par le chercheur avec chaque enseignant à l'IUFM : le premier en décembre 2004, le second en mars, le troisième en juin 2005. Dans ce corpus intermédiaire, apparaissent des différences liées à l'âge (huit stagiaires sont âgés de 23-24 ans, deux de 26 ans, trois de 28 ans), à l'expérience antérieure d'enseignement (quatre stagiaires sur les 13 ont déjà enseigné), à la discipline d'enseignement (cinq stagiaires en Lettres, trois en Mathématiques, deux en Langues vivantes, deux en Éducation Physique et Sportive et un en Physique-Chimie).

Par ailleurs des réponses contrastées ont été retenues pour rendre compte de la diversité du public ${ }^{3}$ : motivations initiales pour l'enseignement, représentations liées au métier (enseignant idéal; qualités essentielles attendues; proximité avec d'autres

2. Au total, l'enquête par questionnaire porte sur 343 enseignants stagiaires, effectuant leur stage en responsabilité en collège et en lycée. L'étude a permis un suivi de 20 stagiaires, dont 13 enseignent en collège.

3. Cette diversité est explicitée dans la partie 3.1.2. 
activités; finalités de l'enseignement). Enfin, la définition de soi en tant que professionnel a constitué un critère de choix important : si neuf d'entre eux se considèrent en novembre comme des «novices» et deux disent être «submergés» par le travail, un stagiaire se définit comme un "enseignant qui progresse vite», et un dernier "comme un (ex) étudiant ayant encore du mal assumer sa fonction d'enseignant» ${ }^{4}$.

Lors de la deuxième rencontre, en mars, six parmi les 13 stagiaires choisis dans l'étude ont été observés dans une pratique de classe. Sollicités par le chercheur car représentatifs du groupe, ils ont accepté de s'engager dans un dispositif s'accompagnant de deux entretiens; l'un conduit avant, l'autre après la séance observée 5 . Le climat de confiance développé lors de la première rencontre, en décembre, semble avoir été déterminant pour entrer dans ce projet. Pour assurer une plus grande continuité, les entretiens se sont déroulés dans l'établissement, si possible sur le terrain même de l'observation. Ils ont été retranscrits intégralement.

Dans le cadre de cette contribution, les entretiens sont traités à partir d'une analyse de contenus construite à partir de thèmes émergeant des discours, en lien avec la question de l'hétérogénéité au collège. De ce point de vue, nous avons opté pour une posture analytique, dans laquelle le chercheur tente de reconstruire le sens : il «engage un dialogue centré sur le sujet et provoque l'expression de sentiments, de jugements, de récits, d'argumentations dont il sait qu'ils possèdent, pour le sujet, un sens subjectif» (DemaZière \& Dubar, 1997, p. 34).

Ces énoncés sont mis en relation avec les observations de séance, étayées a posteriori par l'éclairage d'un formateur-spécialiste de la discipline. L'apport du formateur permet de situer la pratique dans une logique disciplinaire, au carrefour des compétences construites par le stagiaire et de celles attendues par l'institution ${ }^{6}$.

Au final, l'analyse se réalise à plusieurs niveaux; elle prend en compte les écarts entre conception du cours et mise en œuvre effective, entre pratiques et modes d'explicitation des choix réalisés in fine. Elle s'intéresse à l'action de l'enseignant, aux éventuels incidents critiques et aux moments de tension repérés dans l'heure de classe, du point de vue de l'acteur puis de celui de l'observateur, non spécialiste de la discipline. Le chercheur n'étant pas impliqué dans la validation des stagiaires suivis dans l'étude, la prise en compte des écarts se situe dans une approche compréhensive, visant une coconstruction de sens. Les logiques du dire et du faire se trouvent donc appréhendées à travers la parole du sujet, parole située, fortement liée au contexte de la classe et de l'interaction avec le chercheur.

4. L'ensemble des items correspondant à cette partie du questionnaire sont explicités en annexe.

5. Les différents guides d'entretiens utilisés sont décrits en annexe.

6. Les analyses du formateur-didacticien de la discipline ne seront pas développées dans cette étude. 
En effet, il n'y a pas de réalités objectives distinctes de réalités subjectives, mais des réalités objectivées dans des objets, des espaces, des mots, des manières de faire et de dire (LAHIRE, 1998). Ainsi, les approches méthodologiques complémentaires évitent l'observation d'une seule «scène», celle du discours ou de la pratique, dont les logiques émergentes ne représentent que partiellement la réalité. En croisant les méthodologies, apparaissent des hétérogénéités comportementales inaperçues par les acteurs qui tentent bien souvent, au contraire, de maintenir l'illusion de la cohérence et de l'unité de leur soi. Il s'agit donc d'observer les acteurs dans des «scènes» différentes, loin ou au cour de l'action et de comprendre de quelle manière ils donnent sens à leurs actes.

La complémentarité de ces méthodes d'investigation est apparue féconde pour éclairer l'objet de recherche : les réponses issues du questionnaire mettent en relief les types d'expérience construits avant l'entrée dans le métier et ouvrant sur une relative connaissance des publics jeunes; elles donnent aussi un aperçu des valeurs et représentations sur le métier et des difficultés repérées dans son exercice au quotidien par les acteurs eux-mêmes. Les entretiens offrent la possibilité de saisir chez ces enseignants débutants, les préoccupations liées à l'hétérogénéité des élèves et les stratégies éventuellement mises en ouvre pour y faire face. À ce niveau, les trois vagues successives de recueil ont constitué un réel intérêt heuristique pour la compréhension : 1) des points d'appui trouvés par le stagiaire durant l'année de formation; 2) de son évolution professionnelle inscrite entre valeurs, représentations et registres d'action.

Enfin les observations de classe révèlent les registres réellement investis, parfois de façon inconsciente, pour accompagner les apprentissages des élèves.

\section{Résultats}

La manière dont le groupe professionnel se situe par rapport à l'hétérogénéité des publics scolaires est envisagée, dans le cadre de cette étude, à deux niveaux complémentaires.

\section{1. À l'échelle du groupe professionnel $(\mathrm{N}=141)$}

\subsubsection{Quand les expériences antérieures sont questionnées par un nouveau} contexte institutionnel et humain

Deux tiers des stagiaires disent avoir déjà eu une expérience d'enseignement : cours particuliers (42\%), enseignement à l'étranger (en langues) ou dans des structures associatives (20\%). Une expérience en collège est évoquée dans $24 \%$ des cas (17\% 
en lycée), sans que l'on en connaisse les durées effectives. La même proportion (2/3) mentionne des expériences ouvrant sur des responsabilités auprès d'enfants ou d'adolescents : il s'agit le plus souvent d'aides individualisées de type «aide aux devoirs» (26\%). Par ailleurs, la prise en charge de groupes d'adolescents à travers des tâches d'animation (23\%) ou d'encadrement sportif (18\%) est envisagée comme permettant une approche intéressante des publics «jeunes». Enfin, le fait d'assumer des fonctions de surveillance dans les établissements scolaires (17\%) donne une connaissance plus précise du monde scolaire. Ces expériences, vécues d'abord comme un appui pour prendre en main la classe ou appréhender plus finement les difficultés d'apprentissage, se révèlent rapidement décalées dans un contexte scolaire où tout est différent : normes de la formation et regards croisés portés sur une pratique balbutiante, manque d'implication des élèves par rapport à certains contenus pourtant élaborés avec soin, etc. Tout ceci fait question, notamment lorsque s'y rajoutent des comportements problématiques indiquant de la part des élèves un refus d'entrer dans le jeu scolaire. De plus, le stagiaire pointe la difficulté à transférer des pratiques jugées efficientes dans une relation inter-individuelle (cours particulier, aide aux devoirs) à un contexte de classe où il faut aussi faire avancer le groupe, au détriment de certains élèves qui nécessiteraient un suivi plus individualisé. Ces désillusions peuvent entraîner une certaine culpabilité ou, tout au moins en début d'année, le sentiment de ne pas être à la hauteur de la tâche à accomplir.

3.1.2. Quand la volonté de répondre aux missions du collège se heurte à la réalité des élèves

Les réponses au questionnaire, affinées au cours des entretiens, font émerger un certain nombre d'éléments indiquant les préoccupations liées à la prise en compte de l'hétérogénéité des élèves.

\section{L'image d'un enseignant compétent ${ }^{7}$ : un idéal professionnel partagé}

La question visant à repérer les compétences professionnelles attendues en priorité chez un enseignant met en relief trois caractéristiques dominantes au sein du groupe enquêté. Ainsi un enseignant, c'est d'abord une personne qui : «construit des contenus adaptés à la diversité des élèves» (23\%), «mobilise des savoirs pédagogiques» $(21 \%)$

7. Les différentes rubriques entre guillemets correspondent aux items proposés dans le questionnaire. Seul le premier a été pris en compte dans cette analyse alors que la plupart des questions autorisaient le choix ordonné de trois d'entre eux. 
et «maîtrise les savoirs disciplinaires» (21\%). Cet enseignant possède en outre certaines qualités pour mener à bien sa mission : il est «responsable» $(23 \%)$ ce qui suppose l'adoption d'une posture d'adulte, «stimulant» (22\%) pour enrôler les élèves dans les apprentissages, éveiller leur curiosité et «juste» (19\%), ce qui l'amène entre autre à questionner les modalités d'évaluation et leurs effets sur la motivation des élèves. Rapprocher le métier d'enseignant de celui «d'éducateur» (39\%), «d'acteur» (24\%) et "d'animateur" (11\%) semble indiquer la volonté d'assumer la large mission confiée au collège unique où une grande partie du travail de l'enseignant dépasse la seule transmission de connaissances et nécessite la construction d'un ordre scolaire (DEROUET, 1992). De ce point de vue, les finalités que les stagiaires donnent prioritairement au collège sont de "faire acquérir aux élèves des outils et méthodes réinvestissables» (33\%), de «susciter le plaisir d'apprendre» (18\%), de «donner accès à une culture scientifique, littéraire, corporelle, etc.» (16\%) et de "permettre à chaque élève de construire des bases dans la discipline» $(15 \%)$. On saisit, à travers ces énoncés, une réelle volonté de faire progresser tous les (et chacun des) élèves qui leur sont confiés.

\section{Des difficultés à surmonter pour assumer sa mission}

Pourtant, des difficultés sont avancées : «d'ordre didactique» (40 \%) concernant l'adéquation des contenus aux élèves (choix, progressivité, sens), «d'ordre pédagogique» (36\%) lorsqu'il s'agit de tenir des classes jugées difficiles, de régler des problèmes avec certains élèves, de proposer des dispositifs adaptés, etc. En conséquence, les attentes en terme de formation sont relativement homogènes au mois de novembre : "analyse des pratiques professionnelles à partir de problèmes rencontrés en classe» (38 \%), «échanges avec les pairs» (28\%), «analyses didactiques de situations, séances ou séquences» $(24 \%)$. Dans tous les cas, il s'agit de se donner les moyens de progresser dans l'exercice du métier pour répondre de façon optimale à la diversité des élèves. Ainsi le stage en responsabilité permet prioritairement, au terme de deux mois de pratique, de «réfléchir à des méthodes pour faire apprendre» (30\%), «d'apprendre à construire des contenus adaptés au niveau des élèves» (28\%) et de «mieux se connaître» (réactions, limites, etc.) (20\%) pour s'adapter aux imprévus de la classe et assumer une posture de professionnel.

Les entretiens mettent en relief une perception quasi immédiate d'une hétérogénéité que beaucoup ne soupçonnaient pas en choisissant cette profession. Ce phénomène que tous évoquent dès les premières semaines de stage, provoque une tension permanente entre avancée du programme et prise en compte de la diversité des élèves en terme d'apprentissage, de comportements, de besoins, etc. Cette contribution se centre sur les difficultés d'apprentissage, étant bien entendu que le contexte de classe va induire d'autres types de focalisation chez les enseignants débutants. 
3.1.3. Quand la prise en compte de l'hétérogénéité fait question

\section{Entre prise de conscience et sentiment d'impuissance}

À l'échelle des entretiens réalisés en juin 2005, huit des 13 stagiaires suivis dans l'étude font état de lacunes chez les élèves, face auxquelles ils se disent démunis : difficulté à comprendre ce qui fait obstacle dans l'apprentissage, impression de dispersion quand l'attention s'individualise, sentiment de ne pas utiliser les «bonnes méthodes», désarroi devant les efforts infructueux des élèves, perception des limites aux encouragements verbaux. Denis, stagiaire Maths, exprime son désarroi devant une classe de quatrième dans laquelle : «Il y en a beaucoup qui ont des lacunes. J'en ai quatre qui sont vraiment perdus. Enfin, 6 divisé par 3, Elodie m’a répondu : Monsieur, j’ai rien compris aux divisions! [...] Disons qu'elles ont essayé. Mais quand ça fait 3, 4 ans que tu fais plus rien, enfin, en Maths c'est pas possible quoi!».

\section{Des tentatives réussies impossibles à tenir sur la durée}

Les résultats permettent aussi de repérer chez les cinq autres stagiaires suivis dans l'étude, des propositions expérimentées dans la classe pour tenter de remédier aux difficultés des élèves. Il s'agit de dispositifs particuliers le plus souvent approfondis dans le cadre du mémoire professionnel : aide individualisée pensée "collectivement» en amont du cours pour anticiper d'éventuels obstacles face à une nouvelle notion; médiations entre pairs rendues possibles dans un travail de groupe qui ne peut être systématisé; proposition de méthodes d'apprentissage différentes, motivantes mais ponctuelles : «Il y en a un... il a déjà deux ans de retard... là, ça a été un peu le moteur de son groupe... Et il m'a dit : Madame, pourquoi on fait pas ce travail-là à chaque théorème? C'est génial de travailler comme ça! J'ai dit : c'était très bien ici mais il y a d'autres théorèmes qui n'ont pas cette application-là» (Barbara, stagiaire Maths, classe de quatrième).

Ces tentatives essayées dans la classe, lorsqu'elles réussissent, ouvrent des perspectives aux enseignants qui mesurent alors leur action pédagogique aux satisfactions que peut en retirer l'élève, moins enfermé, pour un temps, dans ses difficultés d'apprentissage. Mais ce sentiment de réussite est immédiatement suivi de réserves sur le côté ponctuel de ces dispositifs, lié aux exigences du programme, à la nécessité d'avancer dans le cours car une sorte de culpabilité envers les plus rapides indique aussi la tension qui est la leur, au moins dans les disciplines plus «sélectives». 


\subsubsection{Quand la complexité semble ingérable : relativiser ou différer}

Face à de multiples sources de difficultés qu'ils ont du mal à prendre en compte dans l'ordinaire de la classe, les stagiaires sont tentés de mettre à distance le problème en évoquant un certain nombre de raisons : turbulences de l'adolescence, faible poids sélectif de la matière, lourdeur des programmes, etc. De plus, le manque d'expérience de l'enseignant peut être l'occasion de différer des images idéales encore hors de portée.

Cet ensemble de réponses plus ou moins adaptées traverse les entretiens menés au cours de l'année et tend à révéler un manque évident de ressources pour faire face à l'hétérogénéité du collège. Pourtant, en s'intéressant particulièrement aux stagiaires sans expérience antérieure en matière d'enseignement, l'observation de classe apporte des éléments complémentaires. Ainsi, pour expliciter les processus en jeu, nous présentons, dans la deuxième partie des résultats, une étude de cas qui invite à passer d'un niveau «macro» (le groupe des 141 stagiaires) puis «meso" (le groupe des 13) à un niveau «micro» interrogeant un acteur dans la réalité de sa pratique professionnelle.

\section{2. À l'échelle de l'acteur inscrit dans un contexte professionnel particulier : le cas d'Anaïs}

Choisir d'étudier le cas d'Anaïs, c'est appréhender un profil dominant au niveau du groupe des 13 stagiaires du point de vue de l'âge, de l'expérience antérieure d'enseignement, de la définition de soi en tant que professionnel. Au-delà de représentations professionnelles partagées avec l'ensemble du groupe, cette stagiaire se distingue sur quelques points par une approche singulière du métier. Dans le niveau de résultats précédent, elle appartient au groupe qui tente de prendre en compte la diversité des élèves sans, pour autant, pouvoir inscrire ces actions dans la durée. En fait, parmi les six enseignants débutants suivis sur le terrain, trois — dont Anaïs - mettent en œuvre des logiques d'action permettant de gérer cette diversité, sans avoir pleinement conscience des effets produits par une telle démarche. Les trois entretiens successifs conduits avec cette stagiaire soulignent les tensions, ajustements, évolutions au cours de l'année sur cette question. 
3.2.1. Des écarts entre valeurs, représentations professionnelles et réalité du contexte d'enseignement

\section{Une certaine vision du métier}

Le questionnaire proposé en novembre 2004 livre quelques éléments pour caractériser Anaïs. Agée de 23 ans, sans expérience d'enseignement mais fille d'enseignants, cette stagiaire d'Anglais a choisi très tôt son orientation professionnelle; elle évoque une "passion pour la discipline» et se dit sensible aux «conditions de travail» plutôt attrayantes. L'enseignant idéal «mobilise des savoir-faire, des savoirs disciplinaires, réfléchit sur ses pratiques et analyse leurs effets». Il doit être «méthodique, stimulant et sérieux». Les métiers les plus proches de celui d'enseignant sont ceux «d'éducateur, d'acteur et de psychologue». "Donner accès à une culture» constitue la première finalité de l'enseignement de l'anglais. En novembre, cette stagiaire évoque des «difficultés de gestion de classe», notamment de certains élèves perturbateurs, et «d'adéquation des contenus aux caractéristiques des élèves».

\section{Premiers constats, premières questions}

Le premier entretien (décembre 2004) révèle un écart important entre motivations initiales pour ce métier (enseigner pour communiquer, pour offrir une ouverture culturelle) et réalité de la pratique, surtout dans l'une des deux classes de troisième dont elle a la charge. Certains élèves la déconcertent : de niveau très faible, peu engagés dans les apprentissages, ils ne semblent pas saisir la finalité de ce qu'ils font. Cet ensemble d'éléments favorise les décrochages et les comportements d'indiscipline : «c'est très dur... je n'arrive pas à faire ce que j'avais prévu comme avec l'autre classe. Donc j'essaie de mener à bien dans l'heure au moins un minimum de choses... D'un autre côté, je peux pas suivre à leur place quoi. Donc c'est vrai que j'essaie des fois quand même de leur fournir un contenu... je me dis : si je suis leur rythme, on n'y arrivera jamais».

Face à ce constat, la question du "comment faire» se pose rapidement. Anaïs cherche des appuis à différents niveaux : le conseiller pédagogique, les collègues de l'établissement, les formateurs IUFM, et se donne intuitivement plusieurs pistes à investir.

Lors de l'entretien réalisé en décembre, elle évoque des questions liées au processus enseignement/apprentissage : comment s'adapter aux caractéristiques des élèves sans perdre de vue les objectifs? Faut-il valoriser leur compréhension au risque de délaisser certains ancrages linguistiques? Comment donner des repères pour que tous puissent s'investir dans le travail et progresser? Dans la pratique observée se dessinent des axes prioritaires que les regards croisés du chercheur et du formateur vont venir confirmer. 


\section{Une observation contextualisée}

La séance se déroule avec une classe de troisième constituée de 14 élèves relativement calmes, dans un établissement rural. Dans l'entretien qui précède l'observation, l'enseignante explique son choix de travailler sur une Nouvelle de R. Dahl. Pour dépasser la seule présentation de l'auteur dans une unité du livre, elle décide d'aborder directement l'un de ces écrits. En même temps, consciente que ce travail sera difficile et préoccupée par une absence durant la semaine suivante en raison d'un voyage scolaire, elle envisage de leur fournir une méthodologie permettant à chacun de travailler de façon autonome pendant son absence. Cette perspective l'a engagée dans une préparation très élaborée. Pour l'heure, l'objectif de cette séquence est clairement énoncé : «que les élèves se rendent compte que, même avec l'essentiel, on peut comprendre un texte». L'analyse a posteriori du formateur-didacticien confirme des objectifs ambitieux, des aspects didactiques et pédagogiques bien maitrisés et souligne une forte centration sur les apprentissages des élèves. Cet ensemble de données met en relief une réelle articulation entre réflexion et action, constitutive d'une professionnalité en construction.

\subsubsection{Des transactions pour appréhender la pratique dans sa complexité}

L'entretien post-observation conduit par le chercheur s'inscrit dans une approche compréhensive. Ce moment d'interaction permet de faire émerger des représentations et valeurs fortes qui organisent la pratique, notamment à partir des points de tension repérés soit par l'enseignant, soit par le chercheur. Deux principes d'action apparaissent, orientant le processus d'enseignement-apprentissage.

\section{Premier principe : favoriser les apprentissages dans la classe}

Dans cette optique, chacun est invité à s'exprimer : la distribution des prises de parole montre une connaissance précise des élèves et des stratégies adaptées aux ressources identifiées par l'enseignante : «Adeline, Laura : je les laisse parler quand elles lèvent le doigt et je vais aussi m'appuyer sur elles quand c'est un peu plus difficile... Guillaume, qui a du mal mais qui est très volontaire, je vais plutôt lui faire faire des activités de répétition ou de création quand c'est des énoncés simples. Donc bon, au gré de leurs capacités, tout ça, je vais essayer d'interroger en fonction». Apparaît donc à l'observation une volonté d'encourager, de valoriser de façon optimale chacun des élèves. On repère par ailleurs le souci de donner du sens aux propositions pédagogiques en assurant à chacun la possibilité de comprendre et d'entrer dans le travail demandé. Cela suppose une contextualisation des savoirs : ce qui a été vu et peut constituer un 
point d'appui ici et maintenant; ce qui se fait et va servir ultérieurement pour un travail en autonomie. Enfin, Anaïs informe précisément sur les attendus en terme d'évaluation en fin de séquence, ce qui semble sécuriser les élèves et renforcer la cohérence de la démarche. Celle-ci s'accompagne d'un certain nombre de repères donnés aux élèves : par exemple le tableau, très structuré, est envisagé comme une aide aux apprentissages : "vocabulaire, correction des exercices à droite... notion, petit tableau récapitulatif, phrase clé à gauche... leçon au milieu, etc.». Par ailleurs, la diversification de supports (documents visuels et sonores) offre à chacun des entrées différentes dans les contenus abordés : «en fait, je cherche ce qui peut apporter une assistance à l'élève. Donc là, par exemple c'est de mettre une grille avec les questions, au lieu de les poser forcément tout le temps à l'oral, parce que ça peut les perturber. Donc, même si je les pose à l'oral, ils ont au moins un support visuel, qui est physique, qui reste là. Donc celui qui a pas chopé la phrase, il la voit, donc il peut prendre son temps. Donc je trouve que ça invite à un peu plus d'autonomie de l'élève». Enfin, l'enseignante s'attache à vérifier la compréhension et l'implication de tous : simplification de passages du texte jugés trop complexes, reformulations par les élèves, jeu sur la variété des propositions. En conséquence, elle n'hésite pas à aller "contre les préconisations" institutionnelles, pour accompagner au mieux ses élèves et les engager, à terme, dans un travail en autonomie : «je préfere quand même leur donner des aides... Pour celui qui vraiment ne comprend rien, je vais lui donner les moyens d'essayer de comprendre... souvent, c'est, en fait, des équivalents en anglais ou des choses comme ça».

\section{Deuxième principe : inscrire son enseignement dans des temporalités différentes}

Dans l'entretien, la nécessité pour Anaïs d'effectuer un travail de préparation en amont revient fréquemment. L'intérêt pour l'enseignante consiste ensuite, sur cette base, à intégrer progressivement, lors des interactions en classe, les réponses effectives des élèves : «avant je me disais : bon, il faudra qu'ils me disent ci... ça, et puis normalement ça devrait aller. Enfin il y a vraiment un fossé quoi... maintenant j'ai plus de matière pour préparer mes cours, pour dire : bon, voilà les réels problèmes d'un élève de collège moyen qui rentre en classe d'anglais». Enfin, on repère la prise en compte conjointe de priorités immédiates et de perspectives à plus long terme. Dépassant la seule échelle de la séance ou de la séquence, elle cherche à construire une cohérence sur l'année. De plus, il lui faut veiller à préparer ses élèves pour un éventuel passage au lycée et, en ce sens, rester vigilante sur la continuité des apprentissages et les exigences attendues au-delà du collège. 


\subsection{Une identité professionnelle en prise avec la diversité des élèves}

\subsubsection{Entre objectifs visés et réalité des pratiques}

En mars, Anaïs refuse de parler d'acquisitions stabilisées : d'une part elle éprouve encore des difficultés dans la deuxième classe dont elle a la charge; d'autre part sa référence reste les pratiques d'experts (en filigrane, celles observées chez le tuteur). Pourtant, elle pointe quelques éléments qui lui donnent satisfaction car repérés comme en évolution : une meilleure organisation et cohérence des séquences, une plus grande concision dans ses interventions et une «meilleure gestion du temps dans le sens où ce sont les élèves qui parlent». Elle pense avoir progressé dans une utilisation plus claire et plus efficace des supports visuels, point sur lequel elle a axé son mémoire professionnel. Enfin les modalités d'évaluation lui semblent mieux maitrisées : «maintenant, je sais mieux où je vais». On repère pourtant, à cette période de l'année, une préoccupation majeure : l'apprentissage effectif des élèves en terme de durée, de transférabilité et d'autonomie. Par ailleurs, à l'approche des bilans, la question des programmes refait surface, de même que les missions du professeur, difficiles parfois à assumer.

\subsection{2. À l'heure des bilans: des questions persistantes}

L'entretien réalisé au mois de juin 2005, après validation de son année, lui donne l'occasion de confirmer des priorités pédagogiques énoncées lors de la première rencontre. Ainsi, malgré l'évocation d'essais de différenciation réussis, notamment une grille de compréhension utilisée à l'oral, qui discrimine l'information et permet à chacun de pouvoir s'exprimer à son niveau de ressources, plusieurs points restent préoccupants pour cette enseignante. Tout d'abord, on repère une gestion de l'hétérogénéité toujours en tension. Le fait de «s'attarder avec ceux qui ont des difficultés» provoque chez Anaïs un sentiment de culpabilité vis-à-vis d'élèves très motivés qui pourraient être davantage sollicités. Par ailleurs, elle revient souvent sur la nécessité pour l'enseignant de s'interroger sur son propre rapport à la langue de manière à appréhender autrement les difficultés des élèves : «apprendre à réapprendre, voir la langue non pas comme une langue qu'on parle mais comme aussi une langue à baliser, avec des règles de grammaire... enseigner c'est pas du tout pareil que maîtriser la langue... se mettre dans la peau des élèves, c'est pas évident!». Progressivement, elle pointe la double contribution nécessaire aux apprentissages : un enseignant qui "y croit», des élèves qui s’engagent. Au fil de l'année, cette enseignante découvre la nécessité de varier les propositions, de créer un climat favorable pour apprendre : «j'ai essayé de faire des choses qui leur plaisaient, bien que j’adhère pas totalement non plus à ça... il faut aussi 
qu'ils se contraignent à certaines choses qui leur plaisent pas forcément. Mais j'essaie quand même d'apporter des choses, de varier les documents, les supports etc., de rendre l'apprentissage le plus agréable possible». Malgré cette détermination, elle se refuse à leurrer ses élèves : des efforts sont indispensables de leur part pour construire des compétences. De son côté, elle s'engage et reste convaincue que chacun peut progresser, s'il s'en donne les moyens. Ce parti pris de l'éducabilité oriente son action et reste une source de motivation importante pour le métier : "Ce qui vaut la peine? De vraiment bien préparer ses cours, d'y mettre du cœur aussi, et euh... Et essayer de toujours viser à ce que ça se passe bien, à je ne sais pas, une participation optimale etc. Enfin de pas partir dans l'idée : ouais mais de toute façon, avec eux ça va pas marcher... Faut toujours garder espoir que, de toute façon... il y en a qui vont en tirer quelque chose, et que ça vaut la peine de se donner à fond!». Enfin, face à des échecs même relatifs, Anaïs éprouve le besoin de relativiser, notamment avec la classe qui lui pose problème et pour laquelle elle n'est pas parvenue à enrôler les élèves dans le travail : "Je pense aussi que l'âge me fait déculpabiliser. Enfin, leur âge et le contexte dans lequel ils sont. Je me dis : bon, il faut qu'ils grandissent aussi... Si je fais de mon mieux, je vais pas trop culpabiliser non plus».

\subsubsection{Se définir entre singularité et sentiment d'appartenance}

Lors de l'entretien réalisé en juin, Anaïs se définit comme une personne patiente qui aime communiquer, cherche à comprendre les difficultés rencontrées par les élèves, montre une certaine tolérance pour désamorcer d'éventuels conflits. Elle revendique la nécessité d'accompagner au mieux les apprentissages, quel que soit le passif de l'élève. Cette posture est partagée avec la majorité du groupe professionnel des stagiaires (Roux-PEREZ 2005 (b)) qui vit très mal les prédictions définitives proférées par certains collègues, surtout en début d'année; elle perdure malgré des moments de découragement liés à la rupture du contrat didactique chez quelques élèves. Par ailleurs, Anaïs évoque le sentiment d'appartenir à un collectif de travail : l'ancrage dans la communauté des enseignants a été facilité par le lieu de résidence choisi dans la petite ville où se trouve le collège : proximité, participation aux activités dans et hors de l'établissement ont largement favorisé son intégration dans l'équipe éducative.

\section{Discussion}

L'analyse du corpus questionne les processus de construction identitaire en jeu chez cette stagiaire et met en relief des formes d'adaptation contextualisées. Pourtant, les différents points évoqués ci-après croisent les préoccupations de 
l'ensemble des stagiaires observées dans leur pratique de classe (ROUX-PEREZ, 2006 (b)). L'analyse du cas d'Anaïs entre en cohérence avec les autres cas analysés selon les mêmes procédures, permettant ainsi de construire a posteriori les univers de sens qui traversent des discours fondés sur une pratique effective; les transactions qui s'y déploient mettent en relief l'émergence d'une professionnalité enseignante en prise avec des contextes professionnels à investir pour assurer au mieux sa mission d'enseignant.

\subsection{L'importance du contexte et l'impuissance relative face à la diversité des élèves}

Dans un contexte favorable (classe observée), Anaïs peut construire de nouvelles compétences professionnelles; elles semblent remises en cause par des élèves de l'autre classe de troisième dont elle a la charge et qui expriment une totale démotivation visà-vis des apprentissages scolaires. De fait, l'enseignante éprouve quelques doutes sur les stratégies développées pour faire progresser ces élèves et reste lucide sur le fait que l'implication de ces derniers dans l'apprentissage est problématique, difficile, surtout quand le contrat didactique est entièrement à (re) construire. L'étude met en relief les effets du contexte dans la construction de la professionnalité et l'appropriation des gestes du métier. Bien que ceux-ci soient en partie remis en cause l'année suivante, le sentiment d'avoir su y arriver, même partiellement durant l'année de formation, permet aux stagiaires de s'engager avec plus de conviction dans l'accompagnement des apprentissages à venir, seul et avec les autres.

\subsection{Se construire dans un sentiment de reconnaissance}

Au terme de l'année, dont elle n'a jamais douté de la validation, les préoccupations de cette enseignante se doublent d'un sentiment de réussite, même partielle, valorisé en formation : les interventions dans le groupe de pairs ont été appréciées, le mémoire professionnel bien reçu et les retours lors des différentes visites encourageants. Ainsi, le stage en responsabilité a été évalué de façon positive par les différents formateurs et son implication soulignée par le chef d'établissement. L'équipe disciplinaire, en l'invitant à échanger un certain nombre de documents de travail, a reconnu ses compétences professionnelles, reconnaissance accrue par la crédibilité accordée à ses remarques lors des conseils de classe. Enfin, le collectif enseignant dans son ensemble a intégré cette jeune collègue qui avait choisi pour son année de stage de résider dans une petite ville éloignée du centre de formation auquel elle était rattachée. Cet ensemble 
d'éléments lui donne le sentiment d'aller de l'avant et de progresser dans l'exercice d'un métier qu'elle a choisi. Si elle pointe les éléments favorables à l'avancée de tous, elle repère aussi des moments plus difficiles qu'il faut apprendre à analyser : remise en cause de son propre rapport aux savoirs, des contenus, des méthodes, jamais de sa mission.

Enfin, on repère une prise de confiance progressive nourrie des nombreuses interactions au sein de la formation (RouX-PEREZ, 2005 (b)), faisant dire en fin d'année à Anaïs - comme à de nombreux stagiaires - qu'elle se considère à présent «comme une enseignante à part entière». Les processus de validation de fin de formation renforcent ce sentiment de reconnaissance et d'appartenance au corps professionnel des enseignants.

\subsection{Assumer un changement de posture}

La continuité biographique est assurée à la fois par la filiation (une mère enseignante d'anglais qui a su l'encourager), les valeurs personnelles globalement en adéquation avec les missions confiées aux enseignants ${ }^{8}$. Durant l'année, une réorganisation des représentations a pourtant été nécessaire pour pouvoir continuer dans cette voie : comment passer de l'enseignement d'une langue «que l'on aime» à l'objectif de «faire apprendre» les élèves, y compris ceux qui ne sont pas passionnés, et avec un minimum de collaboration de leur part? Ceci échappe en partie aux stagiaires, bien que la plupart tentent d'avancer sur ce point. Mais comment accepter ce changement de focale qui remet en cause la "passion pour la discipline» évoquée majoritairement par le groupe lorsqu'on l'interroge sur les motivations initiales dans le choix du métier? En gardant un ancrage très fort sur les pratiques culturelles et linguistiques pour soi, en se donnant comme objectif, à terme, de préparer l'agrégation pour rester en contact avec un certain niveau de connaissances et s'ouvrir la possibilité d'enseigner un jour en lycée.

\subsection{Enseigner au collège : un acte politique?}

Pour trois des six stagiaires suivies sur le terrain et visiblement soucieuses de l'avancée de tous dans les apprentissages, enseigner s'inscrit dans un registre de valeurs fondatrices : ne pas reculer devant la diversité des élèves mais en faire un point essentiel de la réflexion et de l'action. Toutes ont conscience de l'importance du travail en équipe pour assumer au mieux leur mission d'enseignant dans un collège où les règles de

8. Texte sur la Mission du professeur exerçant en collège, en lycée d'enseignement général et technologique ou en lycée professionnel. Bulletin Officiel, 29 mai 1997, nº 22. 
l'apprendre sont fragiles et semblent à reconstruire, chaque jour, dans chaque classe et avec chaque élève. Par ailleurs s'impliquer constitue un acte fort : il s'agit de participer à une culture professionnelle et à une dynamique collective; il s'agit si nécessaire de se battre pour défendre un collège réellement ancré sur ses missions d'éducation, d'instruction et d'ouverture culturelle.

\section{Conclusion}

À ce jour, les résultats permettent de repérer les formes de mobilisation mises en œuvre chez les enseignants débutants face à l'hétérogénéité au collège et aux inégalités qui s'y développent. La prise en compte de la diversité des élèves à laquelle les stagiaires ne sont pas préparés peut devenir un élément important dans la construction identitaire et amener ceux qui le peuvent à gérer, à leur manière, les discordances entre objectifs d'enseignement, conduite de classe et avancée des apprentissages. Ainsi, les observations en situation, appuyées par des discours qui se transforment au cours de l'année, montrent comment s'articulent et se régulent, en contexte, représentations, valeurs et pratiques professionnelles. L'étude met en relief la manière dont les enseignants débutants subjectivent la diversité des parcours scolaires. Subie pour les uns qui parviennent difficilement à tenir la classe, prise en compte partiellement pour d'autres, cette diversité peut aussi conduire à des stratégies d'enseignement repérables dans l'action mais reposant sur des équilibres toujours fragiles. Les solutions envisagées localement, dans l'ordinaire de la classe, rendent compte d'adaptations provisoires dans le parcours professionnel du stagiaire et contribuent au développement d'une identité professionnelle largement tributaire des interactions entre logique des acteurs, logique de formation et contexte de travail (Roux-Perez, 2006 (b)). 


\section{Bibliographie}

BAILlAUQUÈS S. Le travail des représentations dans la formation des enseignants. In : Paquay L., Altet M., Charlier E. \&Perrenoud P. (dir.). Former des enseignants professionnels. Bruxelles : De Boeck, 1996, pp. 41-61.

BARBIER J.-M. De l'usage de la notion d'identité en recherche. Éducation permanente, 1996, n 128 , pp. 11-26.

BLIN JF. Représentations, pratiques et identité professionnelle. Paris : L'Harmattan, 1997.

CHARLIER E. Former des enseignants professionnels pour une formation continuée articulée à la pratique. In : Paquay L., Altet M., Charlier E. \&Perrenoud P. (dir.). Former des enseignants professionnels. Bruxelles : De Boeck, 1996, pp. 97-117.

CHARlot B. Du rapport au savoir. Paris : Anthropos, 1997.

Demazière D. \& Dubar C. Analyser les entretiens biographiques. Paris : Nathan, 1997.

Derouet J.-L. École et justice. Paris : Métailié, 1992.

DUBAR C. La socialisation : construction des identités sociales et professionnelles. Paris : Armand Colin, 1991.

Dubar C. Formes identitaires et socialisation professionnelle. Revue Française de Sociologie, 1992, n 4, pp. 505-529.

Hetu J.-C., LaVoie M. \& BaillaUQUES S. (Éds). Jeunes enseignants et insertion professionnelle. Bruxelles : De Boeck, 1999.

JODELET D. Représentations sociales : phénomènes, concept et théorie. In : Moscovici S. Psychologie sociale. Paris : PUF, 1984, pp. 357-378.

LAHIRE B. L'homme pluriel : les ressorts de l'action. Paris : Nathan, 1998.

LAUTIER N. Psychosociologie de l'éducation, regard sur les situations d'enseignement. Paris : Armand Colin, 2001.

Malglaive G. Enseigner à des adultes. Paris : PUF, 1990.

Moscovici S. La psychanalyse, son image et son public. Paris : PUF, 1961.

RaYou P. \& VAN Zanten A. Enquête sur les nouveaux enseignants. Changeront-ils l'école? Paris : Bayard, 2004. 
ROUX-PEREZ T. Identité professionnelle et modes d'implication privilégiés chez les enseignants d'EPS. Les Sciences de l'Éducation - Pour l'Ère Nouvelle, 2003, vol. 36, $\mathrm{n}^{\circ} 4$, pp. 37-68.

Roux-Perez T. Dynamiques identitaires à l'échelle du temps : une étude de cas chez les enseignants d'Éducation Physique et Sportive. Science et Motricité, 2005 (a), $\mathrm{n}^{\circ}$ 56, pp. 75-96.

RouX-Perez T. Quels processus en jeu dans la construction de l'identité professionnelle chez les enseignants-stagiaires du second degré à l'IUFM? In : Actes du $\mathrm{V}^{\mathrm{e}}$ colloque international Recherche(s) et Formation, 14-16 février 2005 (b). Nantes : IUFM. www.paysdelaloire.iufm.fr.

RouX-Perez T. Construction identitaire des enseignants en formation initiale : entre représentations partagées et éléments de différenciation dans le rapport au métier. In : Biennale de l'Éducation, 11-14 avril 2006 (a). Lyon : INRP. www.inrp.fr.

RouX-Perez T. Construction des compétences professionnelles chez les enseignants débutants : entre logique de formation, logiques des acteurs et effets de contexte. In : Colloque CNAM «Usages sociaux de la notion de compétences», 9 mars 2006 (b). Paris : ENSIETA. www.ensieta.fr/jecompetence.

TAP P. Marquer sa différence. In : Ruano-Borbalan J.C. Lidentité : l'individu, le groupe, la société. Auxerre : Sciences Humaines, 1998, pp. 65-68. 


\section{Annexe 1 \\ Extraits du questionnaire PLC2, novembre 2004}

Quelles sont les principales raisons qui vous ont encouragé à devenir enseignant? - ordonnez de 1 à 3, sans ex-aequo, les trois réponses avec lesquelles vous êtes le plus en accord

. réussite dans cette discipline durant le cursus scolaire

. motivation précoce pour l'enseignement

. envie de transmettre une passion pour la discipline

. conditions de travail attrayantes (temps libre, sécurité de l'emploi, etc.)

. influence de l'entourage (parents, amis)

. rencontre dans le cursus scolaire d'enseignants "convaincants"

. moyen de rester en contact avec des adolescents

. choix par défaut

. autre (préciser)

Selon vous, un enseignant, c'est d'abord une personne qui...

- ordonnez de 1 à 3, sans ex-aequo, les trois réponses avec lesquelles vous êtes le plus en accord

. maîtrise les savoirs disciplinaires

. mobilise des savoirs pédagogiques

. construit des contenus adaptés à la diversité des élèves

. connaît la psychologie des adolescents

. utilise des savoir-faire transmis ou acquis avec l'expérience

- repère et corrige les erreurs des élèves

. s'engage dans des projets collectifs (disciplinaires, interdisciplinaires)

. communique avec les différents partenaires de l'école (CPE, personnel de direction, parents, etc.)

. cherche à évoluer

. trouve le moyen de se ressourcer en dehors de son métier

. réfléchit sur ses pratiques et analyse leurs effets

. réalise les différentes tâches qui lui sont assignées par l'institution

. s'adapte rapidement au contexte du cours pour se centrer sur l'essentiel

. autre (préciser) 
Selon vous, quelles sont les qualités essentielles d'un enseignant?

- ordonnez de 1 à 3, sans ex-aequo, les 3 réponses avec lesquelles vous êtes le plus en accord

. compréhensif

. disponible

. entreprenant

. juste

. méthodique

. original

. responsable

. sérieux

. stimulant

. autre (préciser)

Parmi les activités suivantes, quelles sont celles qui se rapprochent le plus du métier d'enseignant?

- ordonnez de 1 à 3, sans ex-aequo, les trois réponses avec lesquelles vous êtes le plus en accord

. acteur (comédien)

. animateur

. bricoleur

. éducateur

. entraîneur

- guide (accompagnateur)

. militant

. spécialiste

. autre (préciser)

Pour vous, enseigner (votre discipline), c'est prioritairement...

- cochez une seule réponse

- permettre l'acquisition de connaissances solides dans la discipline

- faire acquérir aux élèves des outils et des méthodes réinvestissables

. favoriser l'accès à des valeurs morales, civiques et sociales

. susciter le plaisir d'apprendre

. donner accès à une culture (scientifique, littéraire, corporelle, etc.) plus large

- permettre à chaque élève de construire des bases dans la discipline . autre (préciser) 
Dans votre pratique d'enseignant, quelles sont les difficultés rencontrées à ce jour?

- ordonnez de 1 à 3, sans ex-aequo, les trois réponses avec lesquelles vous êtes le plus en accord

. aucune difficulté

. d'ordre pédagogique (gestion de classe difficile, publics scolaires hétérogènes, etc.)

. d'ordre didactique, concernant l'adéquation des contenus aux élèves (choix, progressivité, sens, etc.)

. liées aux conditions de travail (matériel insuffisant, locaux inadaptés, horaires mal placés)

. liées à l'insuffisance de la formation

- liées aux caractéristiques des élèves de votre établissement (contexte défavorable)

. d'ordre relationnel avec certains enseignants

. liées au statut d'enseignant-stagiaire et au regard porté par les autres (élèves, parents, etc.)

- liées au travail avec le conseiller pédagogique tuteur

. autre (préciser)....................

À ce moment de la formation, vous définiriez-vous plutôt comme :

- cochez une seule réponse

. un enseignant «novice» qui a encore beaucoup à apprendre

. un stagiaire peu reconnu (par les élèves, les parents et au sein de l'établissement)

. un enseignant qui progresse vite

. un enseignant déjà efficace

. une personne qui doute actuellement de son choix professionnel

. un ancien étudiant qui a du mal à «entrer dans la peau» d'un professeur

- une personne submergée par le travail (préparations, conduite de classe, évaluations, etc).

. un stagiaire faisant difficilement le lien entre le stage en responsabilité et les autres aspects de la formation

. autre (préciser) 


\section{Annexe 2 : guides d'entretien}

\section{Guide d'entretien n ${ }^{\circ} 1$ (décembre 2004)}

Retour sur le parcours

Revenir sur la «définition de soi».

L'entrée dans le métier : les premiers jours dans l'établissement et dans la classe?

Comment vous situez-vous dans cette année de formation?

Que pouvez-vous dire de votre pratique actuellement?

Pouvez-vous me parler du métier tel que vous le faites? de votre discipline?

Quels sont actuellement vos points forts, vos points d'appui et vos réussites?

Avez-vous eu des difficultés au départ et si oui, ont-elles évolué?

Quelles formes de travail avec le conseiller pédagogique?

Comment vous sentez-vous intégré dans l'établissement?

Qu'est-ce qui pour vous «vaut la peine» dans ce métier?

Avez-vous d'autres centres d'intérêt, hors enseignement, qui participent d'un certain équilibre de vie?

Au cours de l'entretien, revenir sur les réponses aux questions à choix multiple (explicitation du sens donné par le stagiaire aux différents items)

\section{Dispositif autour de l'observation d'une séance (mars 2005)}

\section{Entretien avant la séance}

- faire le point sur le contexte de l'établissement, sur la classe

- place de la séance dans la séquence ou dans le cycle

. objectifs pour cette séance

. organisation pédagogique prévue

. points de vigilance particuliers

Il s'agit, dans l'observation, sur la base de traces écrites:

- de rendre compte de l'ambiance de la classe

- de pointer les comportements des élèves, l'attitude du prof

. de décrire les différents temps, types de tâches, etc.

. de repérer d'éventuels points de tension pour le stagiaire (du point de vue extérieur) 


\section{Entretien post-séance réalisé immédiatement après observation}

La parole est donnée au stagiaire; dans les relances, le chercheur se centre davantage sur le comment que sur le pourquoi.

Propositions :

- quelle(s) analyse(s) «à chaud» des écarts entre le prévu et le réalisé? - quels ont été les moments clés de la séance?

- y a-t-il eu des points de tension? Tenter d'expliciter les registres de préoccupations.

. comment ont été perçus/vécus les moments repérés par l'observateur comme pouvant être problématiques?

. ce qui vous satisfait, ce que vous adaptez ou laissez momentanément en attente (quel compromis?)

. comment envisagez-vous la suite?

Enchaîner avec l'autre guide d'entretien en centrant davantage sur la formation, avec retours éventuels à la séance

\section{Guide d'entretien $\mathbf{n}^{\circ} 2$ (mars 2005)}

État des lieux à ce jour vis-à-vis de la formation? de la pratique professionnelle?

Avancées du mémoire professionnel?

Centres d'intérêt actuels?

Préoccupations professionnelles?

Points d'appui, ressources pour progresser?

Évolution dans la vision du métier, de la formation, des élèves, etc.?

Acquisitions relativement stabilisées?

Attentes vis-à-vis de la formation?

Et la validation? 


\section{Guide d'entretien $\mathrm{n}^{\circ} 3$ (juin 2005)}

État des lieux à ce jour

Retour en arrière sur la formation et sur le stage : moments forts, périodes de tension, aspects négatifs, jalons, déclics, etc.

«L'aventure» du mémoire professionnel

Bilan sur la pratique : points forts, points à affiner, etc.

Au regard de l'expérience professionnelle vécue cette année, quels savoirs jugez-vous essentiels?

Au terme de l'année, que pouvez-vous dire de l'évolution de la classe, des caractéristiques des élèves?

Qu'avez-vous appris durant l'année qui vous semble important aujourd'hui?

Comment s'est opérée l'intégration dans le groupe professionnel des enseignants, dans l'établissement?

Si c'était à refaire? (ou conseils à un PLC2 qui commence la formation) Plus en amont, que diriez-vous de la réalité du métier à un élève qui voudrait s'orienter dans cette voie?

Ce qui vaut la peine par-dessus tout?

Comment voyez-vous, actuellement, votre mission de professeur? Et l'avenir? 


\title{
Dealing with pupils' differing abilities at school or bridging the gap between theory and practice for student teachers in Institut Universitaire de Formation des Maîtres
}

\begin{abstract}
Second-year probationary teachers at a Teacher Training Institute [IUFM] carry out a training period in a school. The article focuses on the processes involved in the making of a professional identity in student teachers especially when faced with mixed ability classes. The article also highlights the gap between the teacher training standards and professional identity and professional practice.

The findings collected from a questionnaire have been supplemented by three semidirected interviews held in the 2004-05 school year. The interviews were conducted with six student teachers were also observed for effective learning in their classes. The findings emphasise how the student teachers place themselves in relation to the pupils' heterogeneous abilities. A case study shows the solutions that were found to deal with the problem of heterogeneity in every day classes. The strategies that are built are temporary adjustments. They play a major part in the construction of a professional identity that rests upon the relationship between individual conceptions, the teaching standards and the professional context.
\end{abstract}

Key words: Professional Identity. Teacher Training. Strategies. Professional Practices.

\section{Tomar en cuenta la diversidad de los alumnos de colegio: entre discursos y prácticas de los docentes principiantes en Institut Universitaire de Formation des Maîtres}

Resumen : Durante el segundo año de formación en el IUFM (Instituto Universitario de Formacion de Maestros), los profesores cursillistas efectuan una práctica en responsabilidad en un establicimiento escolar. El estudio se centra sobre el proceso de construcción de la identidad profesional de los docentes principiantes, sobre todo cuando en el colegio se destaca la heterogeneidad de los alumnos en los aprendizajes. También trata de las diferencias entre las normas de la profesión, las representaciones de la carrera y las prácticas profesionales. 
Una colección de datos por cuestionario esta completada por tres entrevistas semi-directivas durante el 2004/2005 con seis profesores principiantes del corpus, que por otro lado, fueron observados dando clase. Mas allá de la manera con la cual el grupo profesional se situa con respecto a la diversidad de los públicos escolares, un estudio de caso permite destacar soluciones surgidas localmente en el ordinario de la clase. Estas respuestas dan cuenta de adaptaciones provisionales; contribuyen al desarrollo de una identidad profesional tributaria de las relaciones entre lógicas de actores, lógicas de formación y contexto de trabajo.

Palabras claves : Identidad profesional. Formación. Lógicas de acción. Prácticas. des enseignants-stagiaires en Institut Universitaire de Formation des Maîtres. Les Sciences de l'éducation Pour l'Ėre nouvelle, Élèves, professeurs, parents : perceptions et représentations des rôles, interactions et apprentissages scolaires, vol. 40, $\mathrm{n}^{\circ}$ 4, 2007, pp. 107-134. ISSN 0755-9593. ISBN 978-2-9528025-4-3. 